\title{
Resección transuretral de próstata bipolar vs monopolar: análisis peroperatorio de los resultados
}

\author{
Abascal Junquera JMª, Cecchini Rosell L, Salvador Lacambra C, Martos Calvo R, \\ Celma Domenech A, Morote Robles J.
}

Servicio de Urología. Hospital Universitario Vall d’Hebrón. Barcelona.

Actas Urol Esp. 2006;30(7):661-666

\section{RESUMEN \\ RESECCIÓN TRANSURETRAL DE PRÓSTATA BIPOLAR vs MONOPOLAR: ANÁLISIS PEROPERATORIO DE LOS RESULTADOS}

Objetivo: Analizar a corto plazo las posibles diferencias entre el resector bipolar y el resector monopolar convencional en la cirugía endoscópica de la hiperplasia benigna de próstata.

Material y métodos: Estudio prospectivo randomizado de 45 pacientes. Veintiún pacientes fueron intervenidos con resector monopolar (Storz Ch 26, 30ํ) y 24 con resector bipolar (Olympus ch 26, 30ㅇ).

Resultados: La edad media fue de 69,5 años en el grupo bipolar frente a 67,3 del monopolar; flujo máximo medio previo a la cirugía $(7,7 \mathrm{ml} / \mathrm{seg}$ vs $7,2 \mathrm{ml} / \mathrm{seg})$; volumen ecográfico prostático (39,5 cc vs $42,7 \mathrm{cc})$; volumen de resección (13 gr vs 12,6 gr), y el tiempo de resección medio fue de 39,7 min vs 42,5 min. La capacidad de corte se calificó como notable-excelente en el $90 \%$ de los casos en el grupo bipolar frente a un 50\% del monopolar $(\mathrm{p}=0,01)$. La adherencia de fragmentos al asa de resección se consideró abundante o muy abundante en $0 \%$ vs $60 \%(p=0,01)$. La capacidad de coagulación fue excelente-notable en $25 \%$ vs $75 \%$ ( $p=0,03$ ). No hubo diferencias significativas en cuanto a los días de sondaje $(2,92$ vs 3,1$)$, días de lavado $(1,79$ vs 2,05$)$, días de ingreso (3,63 vs 3,67), disminución HTO $(3,48$ puntos vs 3,32$)$ y Na plasmático $(0,52 \mathrm{mg} / \mathrm{dl}$ vs 1,16$)$ ni en los episodios de retención aguda de orina (sólo 1 paciente en el grupo monopolar).

Conclusiones: En nuestra experiencia, la RTUP con SurgMaster en próstatas menores de 70 gr ofrece unas mejores prestaciones peroperatorias al cirujano (mejor capacidad de corte, menor adherencia de fragmentos) frente a la resección monopolar clásica, con similares resultados postoperatorios.

Palabras clave: Resección transuretral de próstata. Energía bipolar. SurgMaster.

\section{ABSTRACT}

BIPOLAR VERSUS MONOPOLAR TRANSURETHRAL RESECTION OF THE PROSTATE: PEROPERATIVE ANALYSIS OF THE RESULTS

Objective: To analyse the differences in the postoperative period between bipolar and monopolar resection of the prostate in the endoscopic surgery of the benign prostatic hyperplasia.

Methods: 45 patients were prospectively randomized. Twenty-one underwent monopolar resection (Storz Ch $26,30^{\circ}$ ) and 24 underwent bipolar resection (Olympus ch 26, 30\%).

Results: Mean age in the bipolar group was 69,5 years versus 67,3 in the monopolar group; mean flow before surgery $(7,7 \mathrm{ml} / \mathrm{s}$ vs $7,2 \mathrm{ml} / \mathrm{s})$; ecographic prostate volume $(39,5 \mathrm{cc}$ vs $42,7 \mathrm{cc})$; resection volume $13 \mathrm{~g}$ vs $12,6 \mathrm{~g}$ and mean resection time was 39,7 vs 42,5 min. Cut capacity was considered notable-excellent in $90 \%$ of the patients in the bipolar group vs $50 \%$ in the monopolar group ( $\mathrm{p}=0,01)$; adherence of fragments were considered abundant or very abundant in $0 \%$ vs $60 \%$ ( $p=0,01$ ); coagulation capacity was excellent-notable in $25 \%$ vs $75 \%$ $(\mathrm{p}=0,03)$. There were no significant differences on the days of catheterization $(2,92$ vs 3,1$)$, continuous irrigation ( 1,79 vs 2,05$)$, hospitalization $(3,63$ vs 3,67$)$, hematocrite descent $(3,48$ vs 3,32$)$ and plasmatic sodium $(0,52$ vs 1,16), neither on episodes of acute urine retention (only one patient in the monopolar group).

Conclusions: In our experience, TURP with SurgMaster resector in prostate smaller than $70 \mathrm{~g}$ offers better peroperative qualities for the surgeon (better cut capacity, less adherence of fragments) than the monopolar resection, with similar postoperative outcomes.

Keywords: Transurethral resection of the prostate. Bipolar electrosurgery. SurgMaster. 
$\mathrm{L}^{2}$ a resección transuretral de próstata (RTUP) continúa siendo el tratamiento de referencia en aquellos adenomas de próstata cuyo volumen permita resecarlos en aproximadamente una hora. Una de las últimas innovaciones técnicas encaminadas a disminuir la morbilidad de la RTUP es el bisturi bipolar, instrumento que no necesita placa de bisturí, realiza corte y hemostasia al mismo tiempo, y utiliza suero fisiológico como líquido de irrigación ${ }^{1}$.

Otra ventaja que presenta la resección bipolar es que en los casos de resección transuretral por neoplasia vesical, no provoca estimulación del nervio obturador, permitiendo de esta manera resecciones sin peligro en las paredes laterales.

El asa de este tipo de bisturí es similar a las habitualmente utilizadas en la RTUP convencional y se diferencia en que el mismo terminal lleva incluidos el electrodo emisor (asa) y receptor de la corriente, separados ambos por una sección de material de alta resistencia y baja conductancia. Este bisturí necesita que uno de los polos o electrodos esté en contacto con el tejido que se desea resecar $^{2}$.

La posibilidad de trabajar con suero fisiológico en lugar de soluciones hiposmolares (glicina) evita la posibilidad de un sindrome de reabsorción por hiponatremia dilucional ${ }^{2,3}$.

El bisturí bipolar funciona mediante pulsos de corriente que evitan la formación de un arco eléctrico entre ambos polos mientras que el asa no entra en contacto con el tejido. Una vez que el asa entra en contacto con el tejido la corriente se hace continua circulando desde el electrodo activo (asa) al electrodo pasivo, a través del medio de menor resistencia (suero fisiológico); esta corriente continua hace que el asa alcance temperaturas muy altas, poniéndose incandescente y produciendo la vaporización y necrosis coagulativa del tejido con el que entra en contacto ${ }^{2,3}$.

En la actualidad existen tres equipos que utilizan energía bipolar para la RTU: Gyrus Plasmakinetic (PK), Vista Coblation system (ACMI) y SurgMaster (Olympus). Tanto en el caso del PK como en el del SurgMaster se utiliza un asa simple, pero dado que las varillas del asa deben conducir la corriente eléctrica en uno y otro sentido, es decir, desde y hacia el generador, el recubrimiento externo de los extremos de las varillas actúa como un polo y el alambre del asa como otro; entre ambos se intercalan unas cortas barritas de cerámica aislante. El resector Vista presenta un asa doble (dispuestas una delante de otra y separadas entre sí por $2 \mathrm{~mm}$ ), de modo que su proximidad facilita la formación del arco de plasma. Todos ellos utilizan diferentes grados de frecuencia pero siempre menores que los sistemas de energía monopolar (Tabla 1$)^{3}$.

Tabla 1

Detalle sobre los distintos sistemas de energía bipolar

\begin{tabular}{lcc}
\hline & $\begin{array}{c}\text { Frecuencia } \\
\text { operatoria (kHz) }\end{array}$ & $\begin{array}{c}\text { Voltaje del pico de } \\
\text { coagulación (V) }\end{array}$ \\
\hline Gyrus & $320-450$ & $80-100$ \\
ACMI & 100 & $65-115$ \\
Olympus & 350 & 120 \\
Energía monopolar & 500 & $500-800$ \\
\hline
\end{tabular}

El objetivo de este estudio ha sido analizar las posibles ventajas que ofrece la RTUP bipolar con SurgMaster frente a las RTUP monopolar convencional, durante la intervención quirúrgica por hiperplasia benigna de próstata y en el postoperatorio inmediato.

\section{MATERIAL Y MÉTODOS}

$\mathrm{El}$ resector SurgMaster/Olympus consta de dos vainas y utiliza dos terminales de corriente (emisor y receptor) que van acoplados al elemento de trabajo (Fig. 1); no necesita placa de bisturí en el paciente, ya que la energía retorna al generador a través del propio elemento de trabajo. Utiliza suero fisiológico como líquido de irrigación. El generador

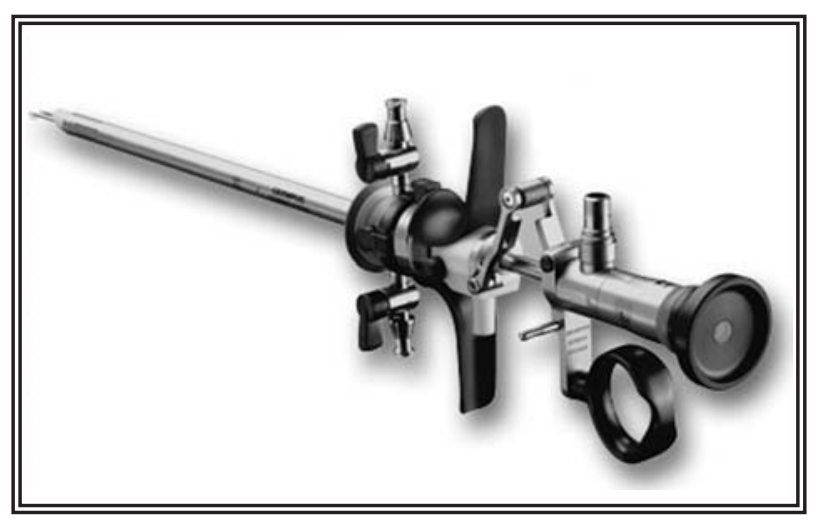

FIGURA 1. Detalle del resector SurgMaster/Olympus. 
SurgMaster tiene gran versatilidad puesto que se puede usar también para obtener energía monopolar o bipolar en cirugía abierta y laparoscópica (Fig. 2). En todos los procedimientos se utilizó una potencia de corte de 250-280 w y de $50 \mathrm{w}$ para la coagulación.

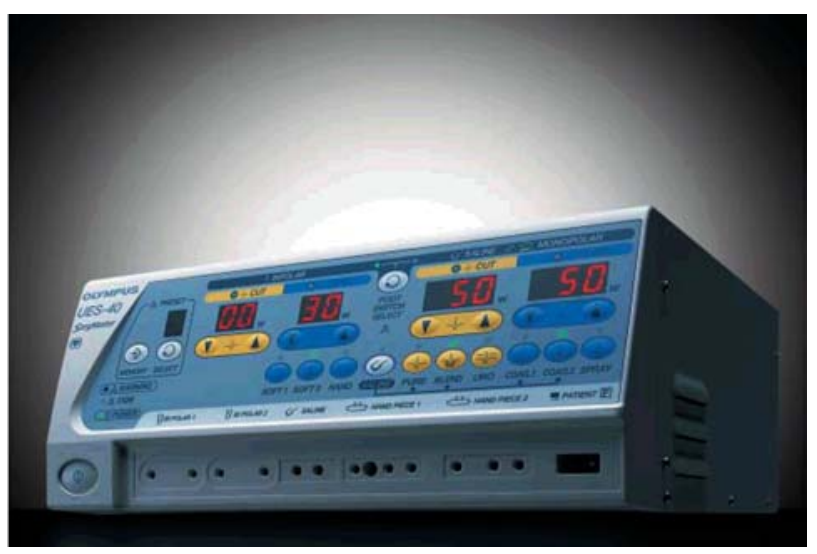

FIGURA 2. Detalle del generador SurgMaster.

Se ha realizado un estudio prospectivo randomizado de 45 pacientes entre marzo y diciembre de 2005 con diagnóstico clínico de hiperplasia benigna de próstata sintomática y volumen prostático ecográfico entre 30 y 70 cc. Veintiún pacientes fueron intervenidos con el resector de doble vaina monopolar Storz ch26 y óptica de $30^{\circ}$ e irrigación continua con solución de glicina. En veinticuatro pacientes se utilizó el resector bipolar SurgMaster de Olympus ch26/30º con irrigación continua de suero fisiológico. En todos los procedimientos se utilizó un asa nueva de resección del mismo tamaño.

Las características de los pacientes asignados a ambos grupos se resumen en la Tabla 2. Se excluyeron todos los pacientes anticoagulados,

Tabla 2

Características de los pacientes de los dos grupos a estudio.

\begin{tabular}{|c|c|c|}
\hline & $\begin{array}{c}\text { Bipolar } \\
\text { Media } \pm \text { desviación } \\
\text { estándar }\end{array}$ & $\begin{array}{c}\text { Monopolar } \\
\text { Media } \pm \text { desviación } \\
\text { estándar }\end{array}$ \\
\hline Edad (años) & $69,5 \pm 9,5$ & $67,3 \pm 9,2$ \\
\hline \multicolumn{3}{|l|}{ Flujo máximo } \\
\hline preoperatorio (ml/seg) & $7,7 \pm 2,1$ & $7,2 \pm 2,5$ \\
\hline Volumen ecográfico (g) & $39,5 \pm 9,8$ & $42,5 \pm 11,6$ \\
\hline Volumen resecado (g) & $13 \pm 8,4$ & $12,6 \pm 6,8$ \\
\hline Tiempo resección (min) & $39,7 \pm 12$ & $42,7 \pm 11$ \\
\hline
\end{tabular}

*en todas las variables $\mathrm{p}>0,05$ con vejiga neurógena, cirugía desobstructiva por adenocarcinoma de próstata o sospecha del mismo y portadores de sonda vesical. En pacientes antiagregados se suspendió la medicación una semana antes de la cirugía. Se recogió urocultivo el día antes de la intervención. Todos los pacientes se sometieron a profilaxis antibiótica con monodosis de gentamicina ajustada al peso del paciente ( 3 $\mathrm{mg} / \mathrm{Kg}$ ) y anestesia raquídea intradural. La sistemática de la resección fue la misma en todos los casos: una vez descartada la presencia de neoformaciones vesicales se realizan primeramente cortes a las 5 y a las 7 horas del huso horario y resección de lóbulo medio, desde cuello vesical hasta veru montanum hasta completar toda la base prostática; posteriormente se resecan los lóbulos laterales y masas apicales. Tras la finalización del procedimiento se realiza revisión hemostática y se extraen fragmentos con bomba de Ellik, colocándose finalmente sonda Dufour de 3 vías ch 20 (globo 20cc) y lavado continuo.

El estudio fue realizado por un total de 6 cirujanos, cada uno de los cuales al acabar la intervención completó un cuestionario para evaluar subjetivamente los siguientes parámetros: capacidad de corte del resector (excelente-escasa), grado de adherencia de fragmentos al asa (muy abundante-escasa), sangrado durante el corte (muy abundante-escaso), visibilidad peroperatoria (excelente-escasa), capacidad de coagulación del resector (excelente-escasa).

Se valoró el tiempo de resección, volumen resecado, días de lavado, días de sonda, días de ingreso, disminución del hematocrito y necesidad de transfusión, disminución del sodio plasmático, y episodios de retención aguda de orina tras retirada de sonda vesical.

El momento de retirada de sonda vesical se estableció por al menos dos cirujanos no conocedores del tipo de resector utilizado con el criterio de ser la orina clara tras 24 horas sin necesidad de lavado vesical continuo. El alta hospitalaria se efectuó después de la confirmación de micciones espontáneas de orina clara.

Los resultados han sido analizados con el paquete estadístico SPSS 12,0 para el sistema Windows, utilizando la t de Student para comparación de variables cuantitativas y la prueba de Chicuadrado para variables categóricas. Las diferencias se consideraron significativas cuando $\mathrm{p}<0,05$. 


\section{RESULTADOS}

La distribución de los dos grupos por edad, volumen ecográfico prostático y flujo máximo previo fue homogénea (Tabla 2). Tampoco se observaron diferencias significativas entre la cantidad de adenoma resecado ( $13 \mathrm{~g}$ vs $12,6 \mathrm{~g}$ ) ni en el tiempo de resección entre ambos grupos $(39,7$ min vs $42,7 \mathrm{~min})$. La velocidad de resección fue también similar (aprox. $1 \mathrm{~g} / \mathrm{min}$ ).

En la Tabla 3 se resumen los resultados de las características peroperatorias según el tipo de resector utilizado. La capacidad de corte se calificó de notable-excelente en el 90\% de los casos en el grupo bipolar frente a un 50\% del monopolar $(\mathrm{p}=0,01)$ (Fig. 3). También resultó estadísticamente significativa a favor del resector bipolar la calificación del grado de adherencia de fragmentos al asa de resección, que se definió como abundante o muy abundante en el 60\% de los casos del grupo monopolar ( $\mathrm{p}=0,01)$ (Fig. 4). Por el contrario, obtuvimos resultados significativos a favor del resector monopolar a la hora de analizar la capacidad de coagulación, que fue calificada como notable-excelente en el $75 \%$ vs $25 \%$ $(\mathrm{p}=0,03)$ (Fig. 5). El sangrado durante la intervención fue calificado de escaso de manera similar en ambos grupos, al igual que la visibilidad peroperatoria de buena-notable sin encontrase diferencias significativas.

Las variables analizadas en el postoperatorio se resumen en la Tabla 4. No se observaron diferencias estadísticamente significativas en cuanto a los días de lavado (1,79 vs 2,09$)$, días de sonda vesical $(2,92$ vs 3,1$)$ ni días de ingreso hospitalario $(3,63$ vs 3,67$)$. Tampoco encontramos diferencias en cuanto a la disminución del hematocrito

Tabla 3

Resultados de las variables peroperatorias en ambos grupos

\begin{tabular}{lccc}
\hline & $\begin{array}{c}\text { Bipolar } \\
\text { \% }\end{array}$ & $\begin{array}{c}\text { Monopolar } \\
\text { \% }\end{array}$ & $\mathbf{p}$ \\
\hline $\begin{array}{l}\text { Capacidad de corte } \\
\text { (notable-excelente) }\end{array}$ & 90 & 50 & 0,01 \\
$\begin{array}{l}\text { Adherencia de fragmentos } \\
\text { (abundante-muy abundante) }\end{array}$ & 0 & 60 & 0,01 \\
$\begin{array}{l}\text { Capacidad de coagulación } \\
\text { (excelente-notable) }\end{array}$ & 25 & 75 & 0,03 \\
$\begin{array}{l}\text { Sangrado (escaso) } \\
\text { Visibilidad (buena-notable) }\end{array}$ & 88 & 90 & $>0,05$ \\
\hline
\end{tabular}

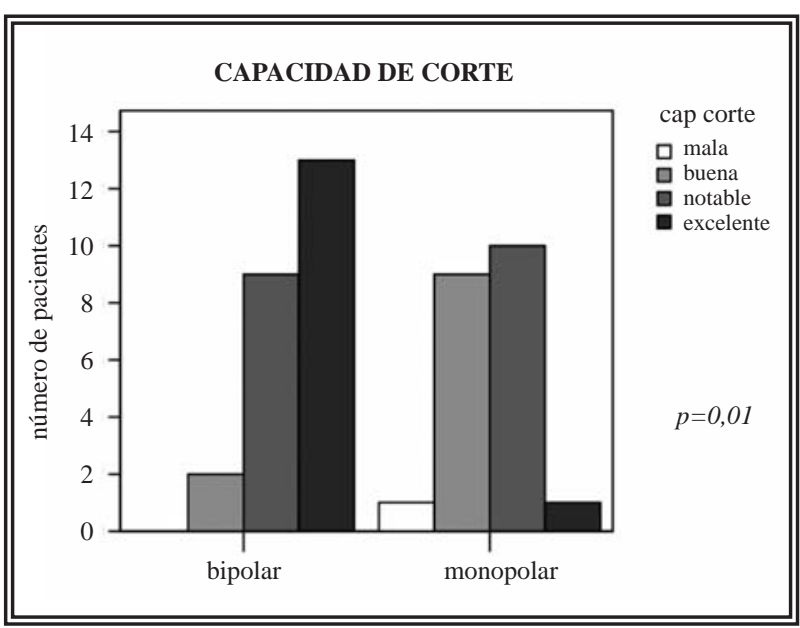

FIGURA 3. Calificación de la capacidad de corte en ambos grupos.

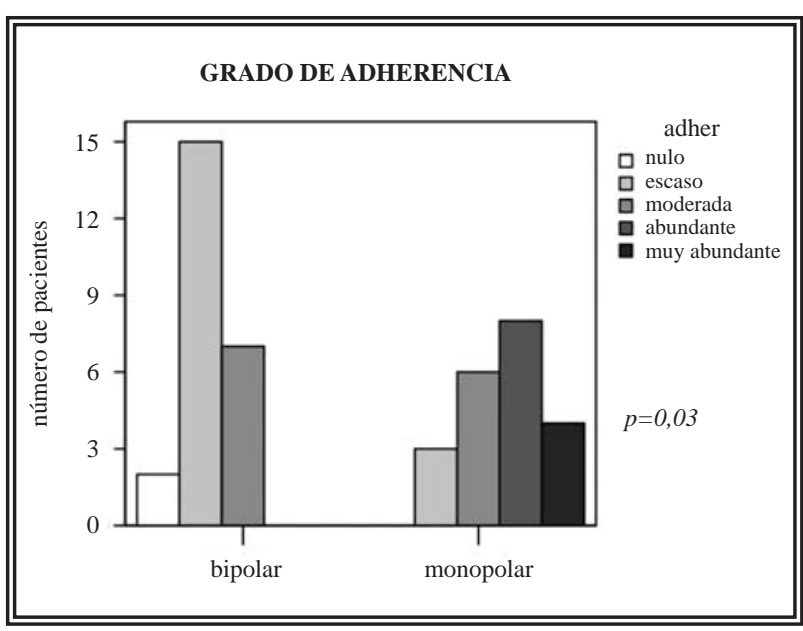

FIGURA 4. Calificación del grado de adherencia en ambos grupos.

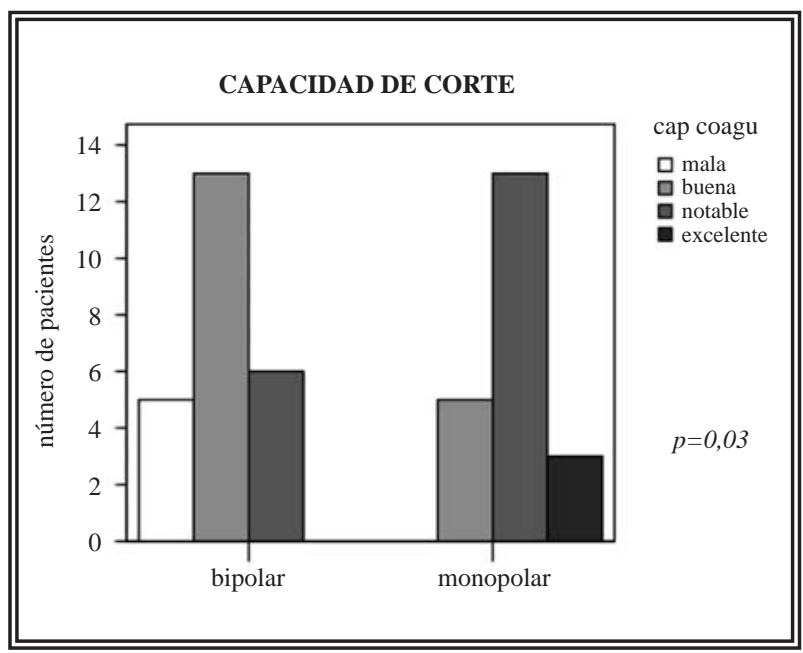

FIGURA 5. Calificación de la capacidad de coagulación en ambos grupos. 
Tabla 4

Resultados de las variables postoperatorias en ambos grupos

\begin{tabular}{lcc}
\hline & $\begin{array}{c}\text { Bipolar } \\
\text { Media } \pm \text { desviación } \\
\text { estándar }\end{array}$ & $\begin{array}{c}\text { Monopolar } \\
\text { Media } \pm \text { desviación } \\
\text { estándar }\end{array}$ \\
\hline Sonda vesical (días) & $2,92 \pm 0,5$ & $3,1 \pm 0,5$ \\
Lavado (días) & $1,79 \pm 0,4$ & $2,01 \pm 0,6$ \\
Ingreso hospitalario (días) & $3,63 \pm 0,5$ & $3,67 \pm 0,5$ \\
Disminución sodio (mg/ml) & $0,52 \pm 2,1$ & $1,16 \pm 3$ \\
Rao & 0 & 1 \\
Transfusión & 0 & 0 \\
\hline
\end{tabular}

*en todas las variables $\mathrm{p}>0,05$

tras la cirugía $(3,48$ puntos vs 3,32 puntos) ni del sodio plasmático $(0,52 \mathrm{mg} / \mathrm{dl}$ vs $1,16 \mathrm{mg} / \mathrm{dl})$. Ningún paciente fue subsidiario de transfusión sanguínea postoperatoria ni se dieron casos de síndrome de reabsorción por hiponatremia dilucional. Sólo un paciente en el grupo del resector monopolar sufrió episodio de retención aguda de orina tras retirada de sonda vesical $(\mathrm{p}>0,05)$.

\section{DISCUSIÓN}

A pesar de los diversos avances en terapias farmacológicas y mínimamente invasivas para el tratamiento del síndrome del tracto urinario inferior, la resección transuretral de próstata continúa siendo el tratamiento quirúrgico de referencia para la hiperplasia benigna de próstata $^{1}$. Aunque este procedimiento se asocia a una mortalidad muy baja (menos del 0,25\%), existen sin embargo dos complicaciones peroperatorias que todavía no son infrecuentes: la pérdida sanguínea (tasa de transfusión entre el 2,5\%-8,6\%) y el síndrome de reabsorción del líquido de irrigación con hiponatremia dilucional $(2 \%$ si el tiempo de resección es mayor a 90 minutos; $0,5 \%$ si es menor de 90 minutos) ${ }^{4}$.

Los nuevos equipos de resección con energía bipolar intentan evitar esta morbilidad, realizando corte y hemostasia al mismo tiempo y utilizando suero fisiológico como líquido de irrigación continua. En este trabajo presentamos nuestra experiencia con el resector SurgMaster (Olympus), que hasta el momento es la única referencia en la literatura ya que otros grupos han publicado resultados con otro tipo de resectores bipolares ${ }^{2,5,7}$ o técnicas diferentes (electrovaporización) $)^{6,8}$.
En primer lugar hemos analizado ciertas cualidades peroperatorias del tipo de resector. Una buena capacidad de corte del resector protege frente a posibles perforaciones de la cápsula prostática por insistencia inapropiada en el corte debido a la no respuesta del asa en un momento puntual de la intervención. El grado de adherencia de fragmentos de adenoma al asa de resección también puede influir en la calidad y duración de la cirugía; estas dos características fueron calificadas positivamente a favor del resector bipolar, mientras que la capacidad de coagulación resultó estadísticamente significativa a favor del resector monopolar. El hecho de que la energía bipolar ofrezca corte y coagulación al mismo tiempo, probablemente disminuye el uso de la coagulación pura. Estas calificaciones subjetivas fueron completadas por seis cirujanos diferentes.

No obtuvimos diferencias significativas en el tiempo de sondaje entre ambos grupos (2,92 días vs 3,1 días). De Sio et al. ${ }^{5}$ coinciden con nuestros resultados siendo el tiempo medio de cateterización de 72 horas.

No se objetivó ningún episodio de retención aguda de orina (RAO) en el grupo de la resección bipolar. En cambio, Fung et al. ${ }^{6}$ objetivan 7 episodios de RAO de un total de 29 pacientes (24\%) tras retirada de sonda vesical a las 24 horas de la intervención. Otro grupo ${ }^{7}$ comunicó un $12 \%$ $(3 / 25)$ de necesidad de recateterización tras una media de duración de sondaje de 1,8 días.

Starkman et al. ${ }^{7}$ comunican en un total de 43 pacientes, un tiempo medio de sondaje en el grupo bipolar de 1,8 días frente a 3,2 del grupo monopolar $(\mathrm{p}=0,12)$ y una estancia hospitalaria media de 1,2 días y 2,1 días, respectivamente $(\mathrm{p}=0,11)$. Sin embargo, De Sio et al. ${ }^{5}$ demuestran diferencias estadísticamente significativas en cuanto a la duración del sondaje (72 horas vs 100 horas), tiempo de lavado vesical continuo (30 horas vs 52 horas), y estancia hospitalaria $(78,2$ horas vs 107 horas) a favor del grupo bipolar. Este grupo coincidió con nuestros resultados al no haber diferencias estadísticamente significativas en cuanto al tiempo de resección, gramos de resección, disminución del hematocrito y del sodio plasmático (no se objetivó ningún episodio de síndrome de reabsorción en ninguno de los dos grupos). 
Valdivia et al. ${ }^{9}$ publicaron su experiencia con el PK de Gyrus en un total de 100 pacientes, 51 de los cuales englobaron el grupo bipolar; obtuvieron diferencias estadísticamente significativas a favor del grupo PK en el tiempo operatorio (55,2 min vs $62,8 \mathrm{~min})$, velocidad de resección $(0,92$ $\mathrm{g} / \mathrm{min}$ vs $0,7 \mathrm{~g} / \mathrm{min}$ ) y hospitalización (5,6 días vs $7,4)$.

En nuestro estudio, aunque todas las variables que se analizaron en el postoperatorio (Tabla 4) no demostraron significación estadística, sí que se objetivaron valores constantemente favorables al resector bipolar. Teniendo en cuenta que se trata de un tamaño muestral pequeño se insinúan ciertas ventajas a favor del resector bipolar que aumentando el número de pacientes pueden constatar diferencias estadísticamente significativas. También es importante reseñar que en el diseño del estudio se excluyeron próstatas de más de 70 g, donde las diferencias entre ambos resectores probablemente sean aún más acusadas, pues se trataría de los casos que precisarían más tiempo de resección.

\section{CONCLUSIONES}

En nuestra experiencia, la RTUP con SurgMaster en próstatas menores de 70 gramos, ofrece unas mejores prestaciones peroperatorias al cirujano (mejor capacidad de corte, menor adherencia de fragmentos) frente a la resección monopolar convencional, con similares resultados postoperatorios a corto plazo.

\section{REFERENCIAS}

1. Wasson JH, Reda DJ, Bruskewitz RC, Elinson J, Keller AM, Henderson WG. A comparison of transurethral surgery with watchful waiting for moderate symptoms of benign prostatic hyperplasia. The Veterans Affairs Cooperative Study Group on Transurethral Resection of the Prostate. N Engl J Med. 1995;332(2):75-79.

2. Barrero R, Sánchez JM, Blasco B, Lázaro J, Valdivia JG. Resección transuretral de próstata con bisturí bipolar. Actas Urol Esp. 2004;28(2):147-151.

3. Smith D, Khoubehi B, Patel A. Bipolar electrosurgery for benign prostatic hyperplasia: transurethral electrovaporization and resection of the prostate. Curr Opin Urol. 2005;15(2):95-100.

4. Doll HA, Black NA, Flood AB, McPherson K. Mortality, morbidity and complications following transurethral resection of the prostate for benign prostatic hypertrophy. J Urol.1992;147(6):15661573.

5. De Sio M, Autorino R, Quarto G, Damiano R, Perdona S, De Lorenzo G, et al. Gyrus bipolar versus standard monopolar transurethral resection of the prostate: a randomized prospective trial. Urology. 2006;67(1):69-72.

6. Fung BT, Li SK, Yu CF, Lau BE, Hou SS. Prospective randomized controled trial comparing plasmakinetic vaporesection and conventional transurethral resection of the prostate. Asian J Surg. 2005;28(1):24-28.

7. Starkman JS and Santucci RA. Comparison of bipolar transurethral resection of the prostate with standard transurethral prostatectomy: shorter stay, earlier catheter removal and fewer complications. BJU Int. 2005;95(1):69-71.

8. Dunsmuir WD, McFarlane JP, Tan A, Dowling C, Downie J, Kourambas $\mathrm{J}$, et al. Gyrus bipolar electrovaporization versus transurethral resection of the prostate: a randomized prospective singleblind trial with one year follow-up. Prostate Cancer Prostatic Dis. 2003;6(2):182-186.

9. Valdivia JG, Regojo O, Sánchez JM, Elizalde B, Navarro J, Hijazo I. La resección transuretral con solución salina: un logro tecnológico aún no asimilado por la urología. Arch Esp Urol. 2005;58(4):335-345.

Dr. J.M ${ }^{a}$ Abascal Junquera

Servicio de Urología Hospital Vall d’Hebron Passeig Vall d’Hebron 119-129 - 08035 Barcelona

E-mail: josuvargas@hotmail.com

(Trabajo recibido el 6 de febrero 2006) 